\title{
Comparison of the atmospheric properties above Dome A, Dome C and the South Pole
}

\author{
Susanna Hagelin ${ }^{a, b}$, Elena Masciadri ${ }^{a}$, Franck Lascaux ${ }^{a}$ and Jeff Stoesz ${ }^{a}$ \\ ${ }^{a}$ INAF Osservatorio Astrofisico di Arcetri, Largo Enrico Fermi 5, I-501 25 Firenze, Italy \\ ${ }^{b}$ Department of Earth Sciences, Uppsala Universitet, Villavägen 16, S-752 36 Uppsala, Sweden
}

\begin{abstract}
The atmospheric properties above three sites on the Internal Antarctic Plateau are investigated for astronomical applications calculating the monthly median of the analysis-data from ECMWF (European Centre for MediumRange Weather Forecasts) for an entire year (2005) thus covering all seasons. Radiosoundings extended on a yearly time scale from Dome $\mathrm{C}$ and the South Pole are used to verify the reliability of the analyses in the free atmosphere and to study the wind speed in the first $100 \mathrm{~m}$ as the analysis-data are not optimized for this altitude-range. The wind speed in the free atmosphere is obtained from the ECMWF analyses from all three sites. It appears that the strength of the wind speed in the upper atmosphere in winter is correlated to the distance of the site from the centre of the polar high.

The Richardson number is employed to investigate the stability of the free atmosphere and, consequently, the probability to trigger thermodynamic instabilities above the three sites. We find that, in a large majority of the cases, the free atmosphere over the Internal Antarctic Plateau is more stable than at mid-latitude sites.
\end{abstract}

Given these data we can obtain a ranking of the three sites with respect to wind speed, in the free atmosphere as well as in the surface layer, and with respect to the stability of the atmosphere, using the Richardson number.

Keywords: site testing, atmospheric effects, turbulence

\section{INTRODUCTION}

The summits of the Internal Antarctic Plateau (Dome A and Dome C) might be among the best places in the world for astronomical facilities. The free atmosphere is extremely clear with a low humidity and low levels of turbulence and the optical turbulence appears to be confined to a narrow surface layer ${ }^{1] 3}$ The height of this surface layer is expected to be lower at the summits than at the slopes of the plateau. Measurements show that the surface layer at Dome $\mathrm{C}^{3}$ is $36 \pm 10 \mathrm{~m}$ while the height measured at the South Pole ${ }^{\sqrt[4]{5} \text { (situated at a slope) }}$ is $220 \mathrm{~m}$ or $270 \mathrm{~m}$.

The largest source of turbulence in the surface layer is the near-surface winds. The surface winds are triggered by the sloping terrain in combination with a temperature inversion. 6 The inversion is present for a large proportion of the time in Antarctica. It forms when the incoming solar radiation is less then the radiative emission from the snow surface which result in a radiative cooling of the air closest to the surface. The calmest conditions are found at the summits as the principal cause that triggers the surface winds, the slope of the terrain, is absent here.

This study is an attempt to better characterize these sites using the analysis data from the MARS archive, containing the model data from the GCM (General Circulation Model) of the ECMWF (European Centre for Medium-Range Weather Forecasts). A detailed description of the analyses data can be found in Geissler \& Masciadri (2006)!7 Data has been used for an entire year, 2005 at 00 UTC (unless indicated otherwise), in an attempt to give a statistical characterization of the differences between the sites.

The use of data has the huge advantage of being able to access data from anywhere in the world, and we also have the possibility to access the past as well as the present and to make simulations of the future. However a GCM describes the circulation of the whole Earth and its usually assumed to have a resolution that

E-mail: hagelin@arcetri.astro.it 
is too low to accurately describe the surface layer 7 Studies have also appeared $\sqrt{8}$ that claims the ECMWF data also are valid, with a good degree of accuracy, near the surface. This study ${ }^{8}$ does only treat summer data and it is possible that their conclusion is not valid for all seasons. A further discussion of this topic is presented in section 2, which investigates the difference between ECMWF data and radiosoundings.

In order to study the atmospheric circulation near the surface it is in principle better to use a mesoscale model as their resolution is much finer and their physics scheme usually is non-hydrostatic so it can resolve processes occurring at a smaller scale. However it is well worth the effort to first investigate the limit to which we can rely on the global models. It is fundamental to have a clear picture of the limitations of the ECMWF data as well as to try get the maximum out of the available information that such a product can give us.

This paper is an attempt to quantify the differences of some critical meteorological parameters above three sites of astronomical interest, Dome A, Dome C and the South Pole. (Dome F will also be discussed with regards to a few of the parameters.) Measurements are also available from the South Pole and Dome C, whereas no measurements are yet available from Dome A.

The scientific goals of this paper are:

- To make a detailed comparison of the offset/difference between radiosoundings and the ECMWF analyses of the wind speed and the temperature (the main parameters defining the stability of the atmosphere) near the surface during both winter and summer. This will permit us to quantify the uncertainty between measurements and analyses. The idea is to define the conditions in which the ECMWF analyses can be used, with a good level of accuracy, to describe the meteorological parameters and then use this tool (ECMWF analyses) to characterize a site for which there are no measurements.

- Using data from radiosoundings we will estimate the statistic median values of the wind speed in the first $150 \mathrm{~m}$ at the South Pole and Dome C, from April to November. With this information we can quantify which site shows the better characteristics for astronomical applications.

- Extending the study of Geissler \& Masciadri $(2006)^{7}$ of the wind speed in the free atmosphere of Dome $\mathrm{C}$ also to the South Pole, Dome A and Dome F. All sites are located on the Internal Antarctic Plateau but at different latitude and longitude. (Their locations are indicated in Table 1,) In this way we intend to quantify which site is the best for astronomical applications. The result of this analysis is fundamental to put in the right perspective the potentialities of Dome C. As already been discussed by Geissler \& Masciadri (2006), ${ }^{7}$ in winter the wind speed grows monotonically above $10 \mathrm{~km}$ a.s.l. (above sea level), achieving median values of the order of $\sim 30 \mathrm{~m} / \mathrm{s}$ at $25 \mathrm{~km}$ a.s.l. Such a strong wind might trigger an important decrease of the wavefront coherence time and, as a consequence, the potentiality of these sites might disappear. Therefore it should be interesting to extend this type of analysis to more sites on the plateau to retrieve some general information of the wind speed above the Internal Antarctic Plateau.

- Extending the analysis of the Richardson number done by Geissler \& Masciadri $(2006)^{7}$ for Dome $\mathrm{C}$ to the three sites (South Pole, Dome C and Dome A) in order to investigate which regions and periods that are less favourable for the triggering of optical turbulence and to identify the site with the best characteristics for astronomical applications. This result should represent the first estimate of the potentialities of Dome A and we are therefore able to provide some reliable conclusions about this site even before some measurements are done on the site. The double interest of this study is, first and foremost the result in itself and second the opening of a path to a different approach for a fast and reliable classification of potential astronomical sites.

This contribution is an extract of a more exhaustive analysis by Hagelin et al $\frac{9}{9}$ 


\section{ECMWF ANALYSES VERSUS RADIOSOUNDINGS}

The ECMWF analyses are compared to radiosoundings from the South Pole and Dome C in order to investigate the reliability of the ECMWF analyses over sites on the Internal Antarctic Plateau. The radiosoundings and the analyses used for this comparison are both from 2006. This year was chosen because of the richer sample of available radiosoundings. The comparison of analyses data discussed further on in this paper are from 2005, the reason being that we wish to investigate a homogeneous data set. In February 2006 the ECMWF changed the number of vertical levels from 60 to 91 .

The median of the difference between ECMWF analyses and radiosoundings is calculated for winter (June, July and August) as well as summer (December, January and February). Particular interest is paid to the first $150 \mathrm{~m}$ of the atmosphere as it is important to quantify the reliability of the analyses data in this layer. In the free atmosphere there is a noticeable difference between the radiosoundings from summer and the ones from winter, namely the difference in the maximum altitude they reach. This fact makes it difficult to study the reliability of the ECMWF analyses in the high part of the atmosphere in winter. During this season the balloons frequently explode at altitudes somewhere around $10 \mathrm{~km}$, probably due to the low pressure in the high part of the atmosphere in combination with the very low temperatures. Due to the low pressure the balloon expand and due to the low temperature, much lower than the average summer temperature at the same altitude, the material of the balloon is more fragile and explode easier.

Figure 1 shows the median of the difference in wind speed and temperature between ECMWF analyses and radiosoundings for summer and winter at Dome $\mathrm{C}$. In local summer the median difference in wind speed never exceeds $1 \mathrm{~m} / \mathrm{s}$. Closest to the ground the difference is even smaller, less than $1 \mathrm{~m} / \mathrm{s}$. During local winter the median difference in wind speed in the free atmosphere never exceeds $0.5 \mathrm{~m} / \mathrm{s}$, though the radiosoundings only reach $\sim 10 \mathrm{~km}$ above the ground. The largest median difference is found near the surface, $\sim 3 \mathrm{~m} / \mathrm{s}$ during this season.

The median difference of the absolute temperature in summer is below $2 \mathrm{~K}$ throughout the whole altitude range investigated. Near the surface this difference is of the order of $1 \mathrm{~K}$, closest to the surface it is even smaller. During winter in the high part of the atmosphere the median difference is similar to what is observed in summer, but in the first $100 \mathrm{~m}$ the median difference is significantly larger, more than $6 \mathrm{~K}$ nearest the surface.

Figure 2 shows the same output but calculated for the South Pole. Only the first $150 \mathrm{~m}$ are shown, for a comparison in the free atmosphere we refer the reader to Geissler \& Masciadri (2006)! ${ }^{7}$ Regarding the wind speed the difference at the South Pole is similar to what is observed at Dome C. The wind speed difference remain within $1 \mathrm{~m} / \mathrm{s}$ in the first $150 \mathrm{~m}$ in summer. The analyses show a tendency to overestimate the wind speed near the ground in winter. The median difference of the absolute temperature is similar to what is observed at Dome $\mathrm{C}, \sim 1 \mathrm{~K}$ in summer. Near the ground the ECMWF-analyses are almost $2 \mathrm{~K}$ warmer than the radiosoundings. The same trend is observed also in winter. However during this season the analyses are visible much warmer $(\sim 6 \mathrm{~K})$ than the radiosoundings near the surface.

The statistic uncertainty of the radiosounding data is reported in Hagelin et al. (2008)!9 The same paper also report a study of the comparison of the median value of the wind speed of the radiosoundings in their first point (the most critical point) in the central months of the winter and AWS (Automatic Weather Stations). The values obtained with the different types of measurements agree in an excellent way. However this comparison permitted us to put in evidence a small bias $(\sim 2 \mathrm{~K})$ in the radiosoundings. This decreases the overestimation of the temperature in the ECMWF analyses to 4-5 K.

Summarizing, the wind speed is well reconstructed by the ECMWF analyses, with exception of the surface layer in winter where the ECMWF analyses show a tendency of overestimating with 2-3 m/s. Considering that a typical wind speed at Dome $\mathrm{C}$ in this season is $\sim 3 \mathrm{~m} / \mathrm{s}$ [10 this corresponds to a large discrepancy. The absolute temperature is in general warmer in the ECMWF analyses than in the radiosoundings near the surface in winter, achieving a difference of the order of $\sim 6 \mathrm{~K}$. The wind speed and the temperature show similar trends above the two sites with exception of the absolute temperature in winter. At the South Pole the temperature from the analyses appear warmer in the whole $150 \mathrm{~m}$ while at Dome $\mathrm{C}$ only in the first $20 \mathrm{~m}$. 

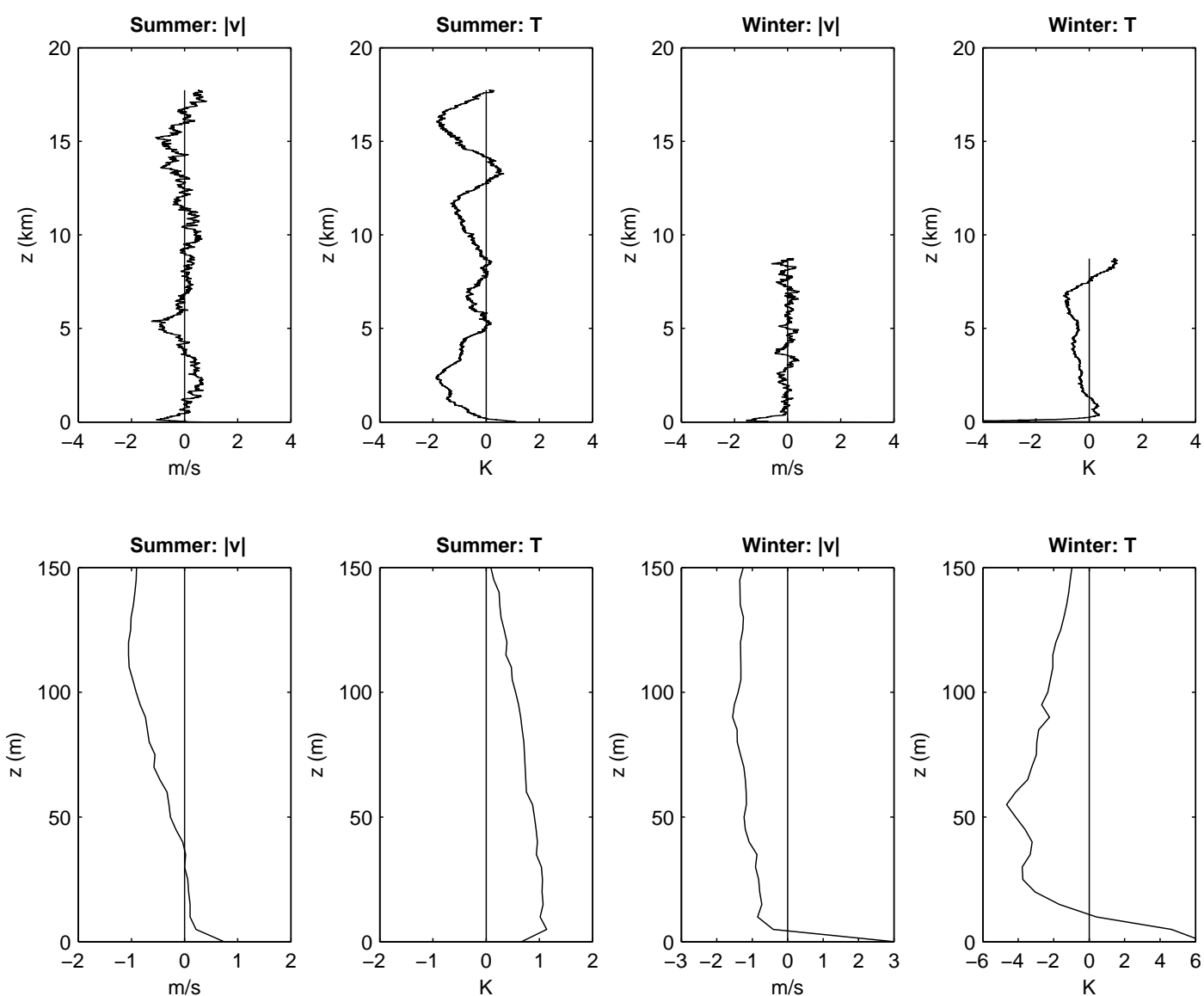

Figure 1. The median of the difference of the absolute temperature and the wind speed between ECMWF analyses and radiosounding for summer (December, January, February) and winter (June, July, August) at Dome C in 2006.
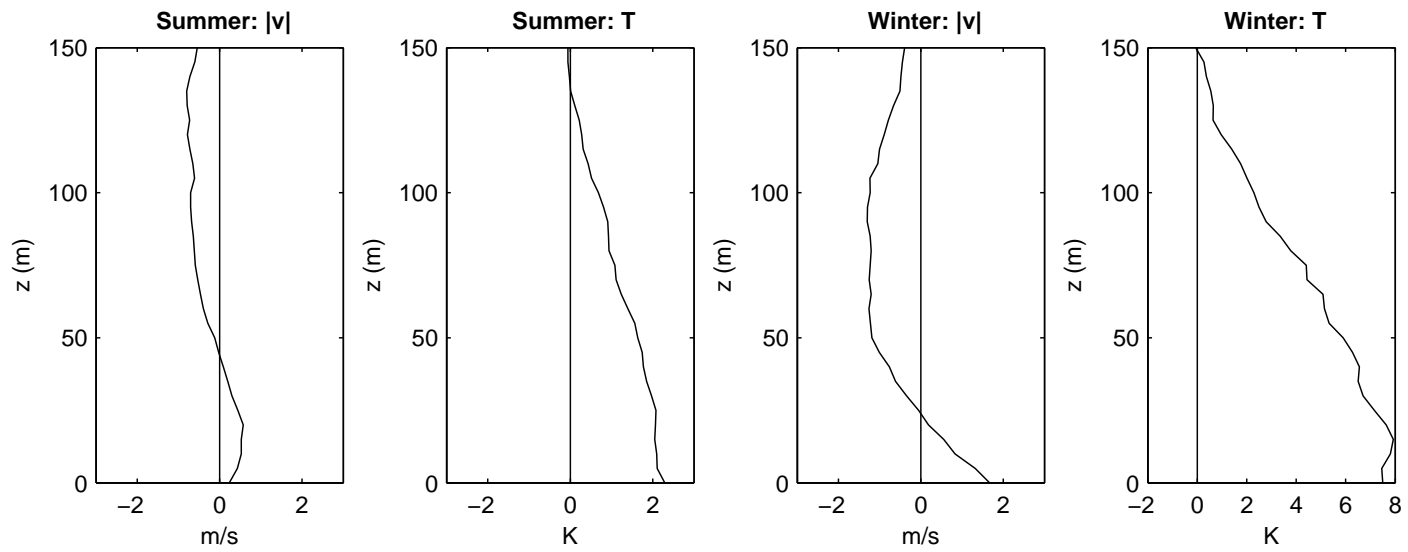

Figure 2. The median of the difference of the absolute temperature and the wind speed between ECMWF analyses and radiosoundings for summer (December, January, February) and winter (June, July, August) at South Pole in 2006. 

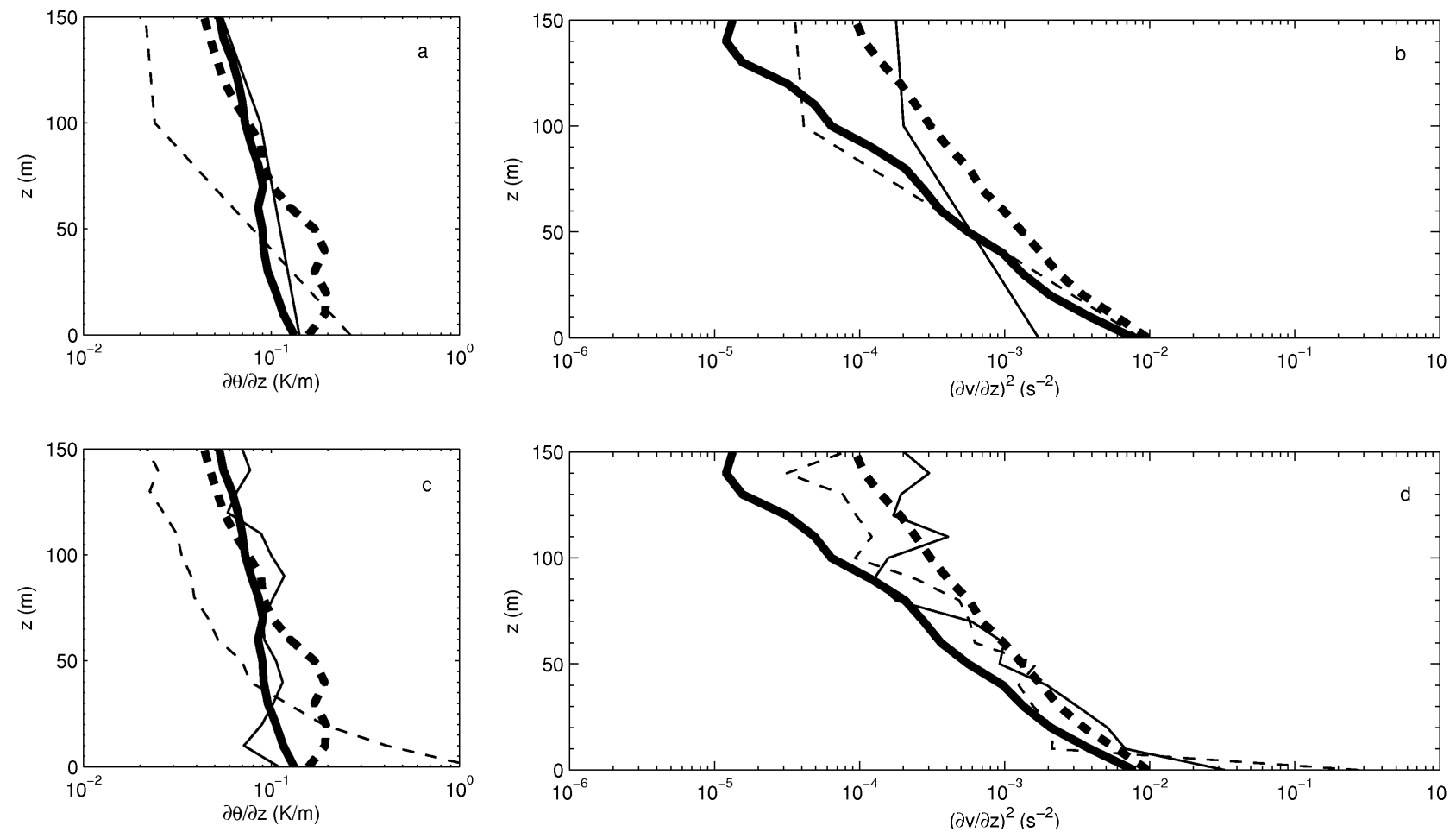

Figure 3. The gradient of the potential temperature and square of the gradient of the wind speed near the surface for Dome C and the South Pole in July 2006. The dashed lines refer to Dome C and the solid lines refer to the South Pole. Thick lines are ECMWF analyses and thin lines are radiosoundings. In the top plots ( $\mathrm{a}$ and $\mathrm{b}$ ) the radiosoundings are interpolated with a step of $100 \mathrm{~m}$, in the bottom plots (c and d) with a step of $10 \mathrm{~m}$.

These conclusions also confirm our doubt expressed in the Introduction concerning the paper of Sadibekova et al. $(2006)^{\sqrt{8}}$ that claimed a good agreement between ECMWF analyses and radiosoundings also in the vicinity of the surface.

In spite of the fact that their study was based on the ERA-reanalyses (a product having a lower resolution than the MARS catalogue used in this study) the agreement between radiosoundings and analyses in their data matches well with our findings that predict a good agreement between ECMWF analyses and radiosoundings in summer. Our analysis, extended to winter, reveals that in this season the agreement is far from being good and the sharp changes in wind speed and temperature closest to the surface measured by the radiosoundings are not well reconstructed by the ECMWF analyses.

In order to provide the most comprehensive and compact comparison of ECMWF analyses and radiosoundings above Dome C and the South Pole near the surface we prefer to change the focus to the two key parameters that define the thermodynamic stability, i.e. the gradients of the potential temperature and of the wind speed. Only a study of the simultaneous systematic effects on both parameters can tell us if it is possible to use ECMWF analyses to quantify the thermodynamic stability in the surface layer.

Figure 3 shows the median gradient of the potential temperature (left) and the square of the gradient of the wind speed (right) in the first $150 \mathrm{~m}$ with a vertical resolution of $100 \mathrm{~m} \mathrm{(a} \mathrm{and} \mathrm{b)} \mathrm{and} \mathrm{of} 10 \mathrm{~m} \mathrm{(c} \mathrm{and} \mathrm{d)} \mathrm{in} \mathrm{the}$ radiosoundings. As expected the radiosoundings show a sharper gradient than the analyses near the surface. The ECMWF analyses are able to identify that the gradients above Dome $\mathrm{C}$ are larger than those above the South Pole. Unfortunately a precise quantification is not possible and, even in the case of the best vertical resolution (c and d), the offset of the analyses with respect to the radiosoundings of the two parameters $\left(\partial \theta / \partial z\right.$ and $\left.(\partial v / \partial z)^{2}\right)$ is not comparable above the two sites. This implies that the ECMWF analyses do not smooth out the potential temperature and wind speed gradients in a similar way above the two sites. 
Knowing that the Richardson number depends on the ratio of $\partial \theta / \partial z$ and $(\partial v / \partial z)^{2}$ we conclude that it is pretty risky to draw any conclusions on a comparative analysis of the Richardson number in the surface layer between different sites calculated with the ECMWF analyses. As a consequence it is possible to retrieve a ranking of the three sites with respect to the thermal and the dynamic stabilities in an independent way, but it is not possible to retrieve a ranking of the three sites with respect to the Richardson number in the surface layer. In the free atmosphere the ECMWF analyses are reliable and such a comparison will be performed in Sec. 5 .

\section{$2.1 \partial \theta / \partial z$ and $(\partial v / \partial z)^{2}$ at the South Pole, Dome C, Dome A and Dome F}

As a consequence of the conclusions in the previous section, the 'thermal' and the 'dynamic' properties are shown independently. The dynamic properties are described by the changes in wind speed with height and the thermal stability is represented by the vertical gradient of the potential temperature. A positive potential temperature gradient is defined as stable condition, the vertical displacement of air is suppressed and so is the production of dynamic turbulence.

The absence of sunlight in the antarctic night and the consequent radiative cooling of the snowy ice surface creates a strong temperature inversion near the surface. The monthly median of the gradient of the potential temperature in the first $150 \mathrm{~m}$ for the four sites is shown in Fig. 4. The analyses data used for this figure are the data from the most stable synoptic hour, i.e. the synoptic hour closest to local midnight, 00 UTC at the South Pole and Dome F and 18 UTC at Dome A and Dome C. These times corresponds to 23 LT (Local Time) for Dome A, 02 LT for Dome C and 03 LT for Dome F. An inversion is always present during winter. In the central months of the winter (June, July, August) Dome A (dark blue lines) shows the most stable stratification, closely followed by Dome F (light blue lines). The inversion above Dome A is from March to August clearly larger than for the other sites and has a very sharp change in the slope of the gradient at about $20 \mathrm{~m}$ above the surface. Dome C (green lines) and the South Pole (red lines) also has a clearly stable stratification but it is lesser than for the other two sites.

During the summer months the stratification is close to neutral, particularly in December. When the stratification is neutral $(\partial \theta / \partial z \approx 0)$ the vertical motion of air is not suppressed but neither is it encouraged and a small perturbation can trigger dynamic turbulence. However, in this season there is a diurnal variation in the antarctic boundary layer where the stratification is close to neutral during the day and during the night an inversion is created. There is a clear difference between the South Pole, that during all summer has a neutral gradient while the domes all show a clear temperature inversion near the surface during the night, as shown in Fig. 4. To draw any conclusion of the differences between the domes is quite risky in this season as the temporal resolution is a little too low.

For the median of the gradient of the wind speed in the first $50 \mathrm{~m}$ above the surface of the South Pole, Dome $\mathrm{C}$ and Dome A we refer the reader to Fig. 5 of Hagelin et al (2008). ${ }^{9}$ The low resolution of the ECMWF analyses makes it difficult to see any clear differences between the sites for this parameter, but almost every month the gradient is largest at the lowest level for Dome A.

\section{RADIOSOUNDINGS: THE SURFACE WIND SPEED}

Above the summits of the Internal Antarctic Plateau the surface winds are expected to be weaker than elsewhere on the Plateau. Fig. 5 shows the median wind speed near the surface measured with radiosoundings at the South Pole (dashed lines) and Dome C (solid lines) from April to November. While it is true that the wind speed at the lowest level is weaker at the summit (Dome C) than at the slope (South Pole), there is a sharp increase in the wind speed above Dome $\mathrm{C}$ in the first few tens of meters. At the height of 10/20 m, from May to November i. e. winter, the wind speed is higher above Dome $\mathrm{C}$ than above the South Pole. Above this height the wind speed at Dome $\mathrm{C}$ is either higher than or very similar to the wind speed at the South Pole.

In the core of the winter (June, July, August) the wind speed above Dome C reaches $8 \mathrm{~m} / \mathrm{s}$ at $20 \mathrm{~m}$ and 9 $\mathrm{m} / \mathrm{s}$ at $30 \mathrm{~m}$. The sharp change in wind speed in the first 10/20 m matches our expectations of a large wind speed gradient. This is a necessary condition to justify the presence of optical turbulence in the surface layer ${ }^{3}$ in spite of very stable thermal conditions (i.e. a positive gradient of the potential temperature). 

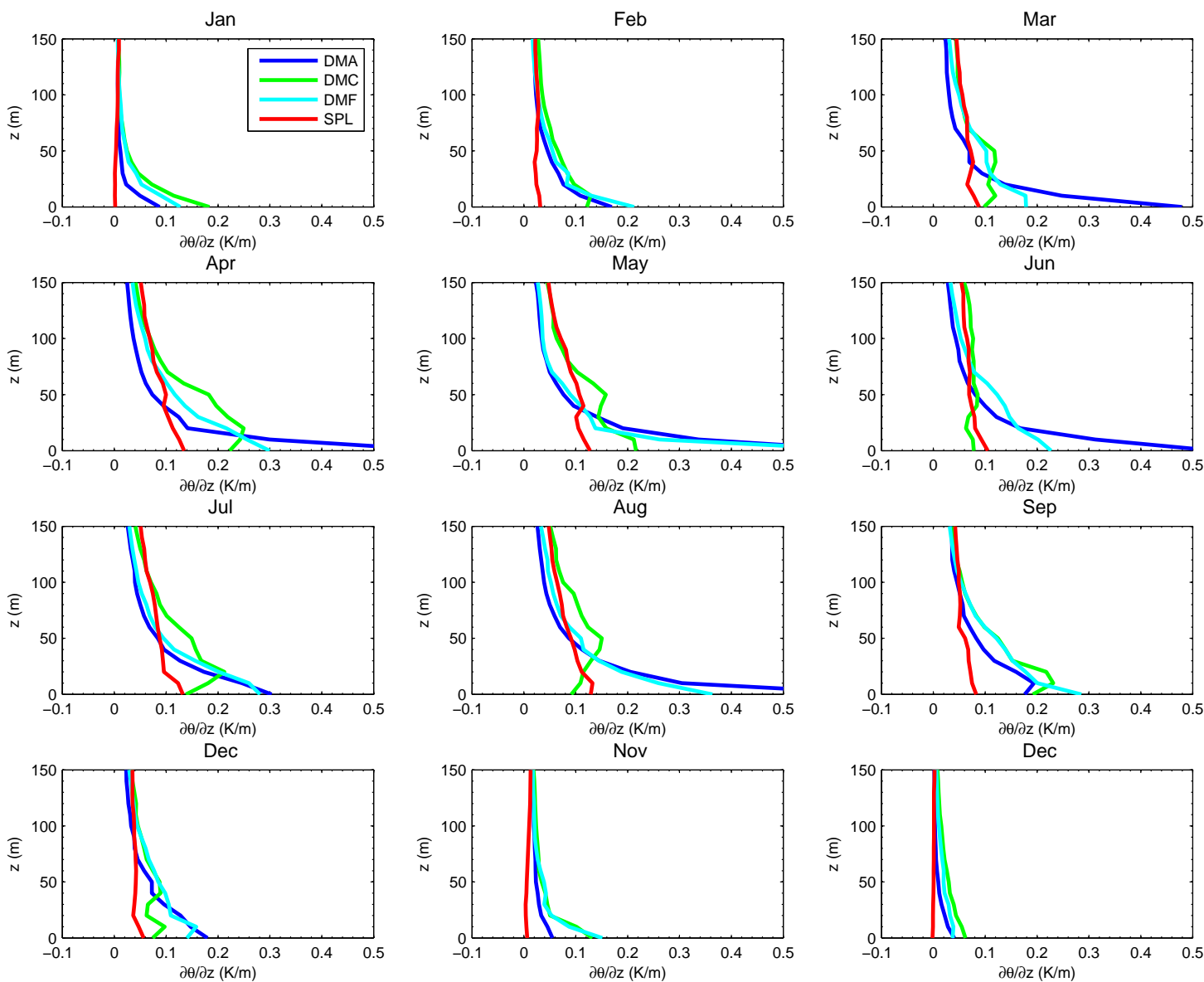

Figure 4. The monthly median of the gradient of the potential temperature for 2005, Dome A (dark blue lines), Dome C (green lines), Dome F (light blue lines) and the South Pole (red lines).

Trinquet et al (2008) $\frac{11}{11}$ using a small sample of radiosoundings in winter, observed a wind speed of $5 \mathrm{~m} / \mathrm{s}$ at $20 \mathrm{~m}$ altitude and only $8 \mathrm{~m} / \mathrm{s}$ at $70 \mathrm{~m}$. Our result, obtained with a complete statistical sample in winter, tells us that their estimate is too optimistic and we should expect a larger wind speed at low altitudes.

Mechanical vibrations in the first $10 / 20 \mathrm{~m}$, that might be derived from the impact of the atmospheric flow, flowing at $8-9 \mathrm{~m} / \mathrm{s}$, on a telescope structure, are probably more critical above Dome $\mathrm{C}$ than above the South Pole. This should be taken into account carefully when designing astronomical facilities.

\section{ECMWF ANALYSES: THE WEAKEST WIND SPEED IN THE TROPOSPHERE AND THE LOWER STRATOSPHERE}

The monthly median of the wind speed, from the ground up to $25 \mathrm{~km}$ a.s.l. for Dome A (dark blue line), Dome C (green line), Dome F (light blue line) and the South Pole (red line) is shown in Fig. 66 During summer the wind speed is quite weak in the whole height interval for all sites. A small maximum is observed at roughly $8 \mathrm{~km}$ a.s.l. From December to March the median wind speed is never larger than $15 \mathrm{~m} / \mathrm{s}$ at any height for any site. As the winter approaches the wind speed in the upper atmosphere increases. Above $10 \mathrm{~km}$ a.s.l. the wind speed increases monotonically and the highest wind speed is more probably found in the higher part of the atmosphere. The rate at which the wind speed increases is not the same above the different sites. The smallest increase rate is observed at the South Pole whereas the maximum rate is found above Dome C. These differences are far from being negligible since at these heights the median wind speed at Dome $\mathrm{C}$ is almost twice that of the other three sites. Geissler \& Masciadri $(2006)^{7}$ found a similar trend of the wind speed at Dome C for the years 2003 and 2004. The trend of the wind speed is therefore confirmed in different years. 

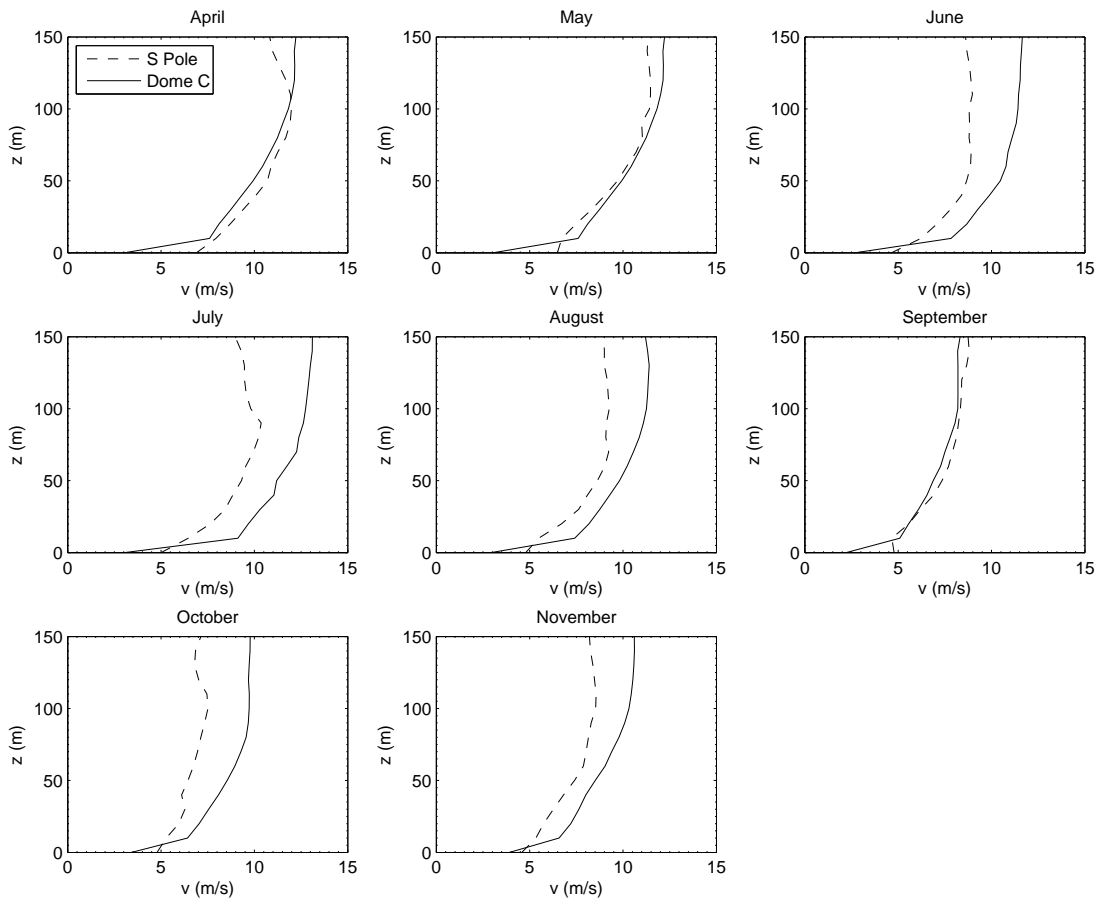

Figure 5. The wind speed near the ground at Dome C (solid lines) and the South Pole (dashed lines), April to November 2006.
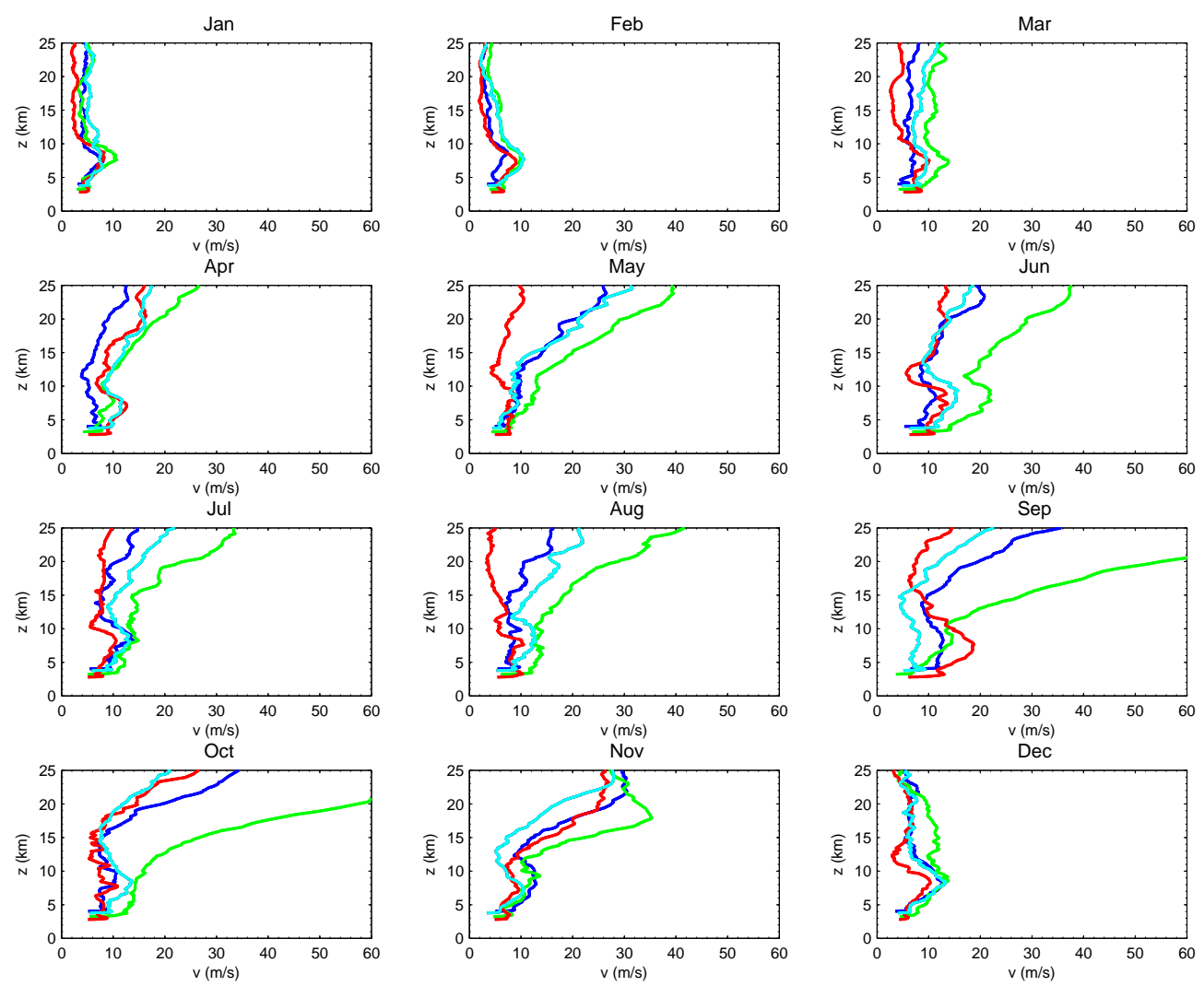

Figure 6. The monthly median wind speed for 200500 UTC for Dome A (dark blue lines), Dome C (green line), Dome F (light blue) and the South Pole (red lines). 
The difference in the slope of the vertical profile of the wind speed in Fig. 6] is most likely related to the special synoptic circulation over Antarctica. The jet stream, that characterize the vertical wind speed profile at mid-latitude sites at the tropopause level, does not form over Antarctica. The large scale circulation is instead dominated by the polar vortex which creates strong high-altitude winds surrounding the polar high in winter ${ }^{6}$ The wind speed increases monotonically above $10 \mathrm{~km}$ (the height of the tropopause). The result observed in Fig. 6 can be explained by the fact that the South Pole is located near the polar high and consequently the influence of the polar vortex is weak at this site. The domes are situated further from the centre of the continent, and thus from the centre of the polar vortex. It is to be expected that the wind speed is larger above these sites. The farther from the polar high (the centre of the polar vortex) the site is, the greater is the strength of the wind speed above the tropopause.

The assumption that the strength of the wind speed in the upper atmosphere is correlated to the distance of the site from the centre of the polar vortex means that if we knew the exact position of the polar high we would have an excellent tool to identify, a priori, the site site with the weakest wind speed above the tropopause during the winter. Unfortunately the polar vortex is not a perfect cone so the centre of the vortex is not located at the same coordinates at different heights. Looking at Fig. 6, it is evident that the South Pole and Dome C respectively have the weakest and the greatest wind speed above the tropopause. The wind speed above Dome $\mathrm{A}$ and Dome $\mathrm{F}$ is mostly comparable and it is more difficult to tell which site has the weaker wind speed. The polar high probably fluctuates in a region located between the South Pole, Dome A and Dome F. (See Fig. 8 in Hagelin et al. $\left.(2008)^{9}\right)$

\section{THE RICHARDSON NUMBER AT THE SOUTH POLE, DOME C AND DOME A}

The Richardson number is an indicator of the stability of the atmosphere,

$$
R i=\frac{g}{\theta} \frac{\partial \theta / \partial z}{(\partial v / \partial z)^{2}}
$$

where $\mathrm{g}$ is the gravitational acceleration $\left(9.8 \mathrm{~m} / \mathrm{s}^{2}\right), \theta$ is the potential temperature and $\mathrm{v}$ is the horizontal wind speed. The atmosphere is considered to be stable if the Richardson number is larger than a critical value, typically 0.25. If the Richardson number is less than the critical number the stratification is classified as unstable. The smaller the Richardson number is the higher is the probability of the triggering of turbulence.

Comparing the Richardson number gives a relative estimate of the probability of the triggering of turbulence above different sites. Fig. 14 of Geissler \& Masciadri $(2006)^{7}$ shows the median of the inverse of the Richardson number $(1 / \mathrm{Ri})$ for Dome $\mathrm{C}$ in the first $20 \mathrm{~km}$ of the atmosphere. However such a result can only give a comparative analysis that necessarily is qualitative. It is possible to identify if one region shows a higher or smaller probability to trigger turbulence than another region but we do not have a reference to quantify these differences nor can we tell whether these differences are negligible or not. In order to investigate this idea further we calculated (Fig. 7) the median of 1/Ri from Dome C (thick lines) and from Mt Graham, Arizona (thin lines), taken as an example of a typical mid-latitude site, for each month during 2005. The inverse of the Richardson is shown instead of the Richardson number because the inverse shows a better dynamic. The smaller $1 / \mathrm{Ri}$ is, the more stable is the atmosphere and the $1 / \mathrm{Ri}$ is smaller above Dome $\mathrm{C}$ than above Mt Graham almost everywhere. This result definitely confirms the method proposed by Geissler \& Masciadri $(2006)^{7}$ to use the $1 / \mathrm{Ri}$ as a tool to rank different sites with respect to the probability to trigger turbulence. During September and October, when the median wind speed at high altitudes is remarkably strong at Dome C (see Fig. 6), 1/Ri is larger than for the mid-latitude site. Consequently this region needs to be carefully monitored as the strong increase in wind speed increases the $1 / \mathrm{Ri}$ and thus the probability to trigger instabilities is larger or comparable to that over a mid-latitude site.

The vertical median profiles of $1 / \mathrm{Ri}$ for the three Antarctic sites (South Pole, Dome C and Dome A) are shown in Fig. 8. During the summer the profiles from the different sites are very similar to each other. The 1/Ri has a maximum at ground level and a smaller peak somewhere slightly above $6 \mathrm{~km}$ a.s.l. and above $\sim 10$ $\mathrm{km}$ the atmosphere is very stable. From April/May the instability above $10 \mathrm{~km}$ increases. At the end of winter (September/October) the instability in the upper part of the atmosphere is even larger than the peak near 6 
$\mathrm{km}$ for Dome A and Dome C. The instability above Dome C is more pronounced than that of Dome A. Note that the wind speed is also larger at Dome $\mathrm{C}$ in this region (Fig. 6). Above the South Pole 1/Ri shows the best conditions (i.e. the atmosphere is most stable) over the whole year.

\section{CONCLUSIONS}

From a first comparison of the atmospheric characteristics of three different sites on the Internal Antarctic Plateau: Dome A, Dome C and the South Pole using ECMWF analyses and radiosoundings we can draw the following conclusions.

The comparison of the ECMWF data to the radiosoundings shows that the ECMWF analyses are reliable above the Internal Antarctic Plateau during all seasons and altitudes except in the first tens of meters. During no season does the difference in wind speed exceed $1 \mathrm{~m} / \mathrm{s}$ above this height. The median difference in absolute temperature is within $2 \mathrm{~K}$ in the same range. The discrepancy of the wind speed in the surface layer is slightly larger, $2-3 \mathrm{~m} / \mathrm{s}$, while the analyses also show a tendency to overestimate the temperature in the lowest level in winter by $\sim 4-5 \mathrm{~K}$. However, one should also remember that during winter the radiosoundings seldom reach an altitude higher than 10-12 km, making it difficult to estimate the reliability of the analysis at high altitudes during the cold season.

Near the surface the estimate of the ECMWF analyses are less accurate and this justify the use of mesoscale models when characterizing the boundary layer. However it is also fundamental to verify that the mesoscale models do provide a better estimate than the General Circulation Models (GCM) in this layer.

The gradient of the potential temperature indicates a stable stratification at all four sites, even if the ECMWF analyses generally underestimate this gradient when compared to measurements from radiosoundings. The stability is particularly strong in winter where Dome A is the site with the steepest gradient, followed by Dome F. The site with the least stable conditions, in this study, is the South Pole.

The median wind speed close to the surface is weaker at Dome $\mathrm{C}$ than at the South Pole during the period investigated (April to November). However, the wind shear at Dome C is much larger than that of the South Pole and so that at 10/20 m the wind speed of Dome C is comparable or larger to the South Pole. Such a strong wind shear in combination with a stable thermal stratification in this layer is most likely the cause of the intense optical turbulence measured in the first tens of meters at Dome C. ${ }^{[3}$ Such a strong wind speed, $\sim 8-9 \mathrm{~m} / \mathrm{s}$ at 10 $\mathrm{m}$, might be a source of vibrations produced by the impact of the atmospheric flow on telescope structures and should therefore be taken into account in the design of astronomic facilities.

In the free atmosphere above $10 \mathrm{~km}$, the polar vortex causes the wind speed to increase monotonically in winter. The increase rate is proportional to the distance of the site from the polar high. This is the reason why Dome $\mathrm{C}$ shows the largest wind speed above $10 \mathrm{~km}$ in winter. At $15 \mathrm{~km}$ altitude the wind speed of Dome $\mathrm{C}$ can easily be almost twice of the wind speed of Dome A or Dome F and even thrice to that of the South Pole. The wind speed above the South Pole is the weakest of the four in all seasons and at all heights.

The monthly median of the inverse of the Richardson number (1/Ri) indicate that the probability to trigger turbulence is larger above a mid-latitude site (for which we have a reliable characterization of the turbulence) than above any site investigated by us at the Internal Antarctic Plateau, except the upper atmosphere at Dome $\mathrm{C}$ during September and October. This is a definite proof that the method proposed by Geissler \& Masciadri $(2006)^{7}$ is reliable. Moreover, using this method to compare our three antarctic sites the $1 /$ Ri-values indicated that Dome A and the South Pole show more stable conditions than Dome C above the first $100 \mathrm{~m}$. This is probably due to the polar vortex that increases the wind speed in the upper atmosphere, which also increases the probability to trigger turbulence. Near the surface it is risky to use estimates of 1/Ri from the ECMWF data as we do not observe an equivalent smoothing effect for the gradients of potential temperature and wind speed.

This study allowed us to draw a first comprehensive picture of the atmospheric properties above the Internal Antarctic Plateau. In spite of the generally good conditions for astronomical applications, Dome $\mathrm{C}$ does not appear to be the best site with respect to the wind speed, in the free atmosphere as well as in the surface layer. All the other three sites show a weaker wind speed in the free atmosphere. The estimates of the surface 

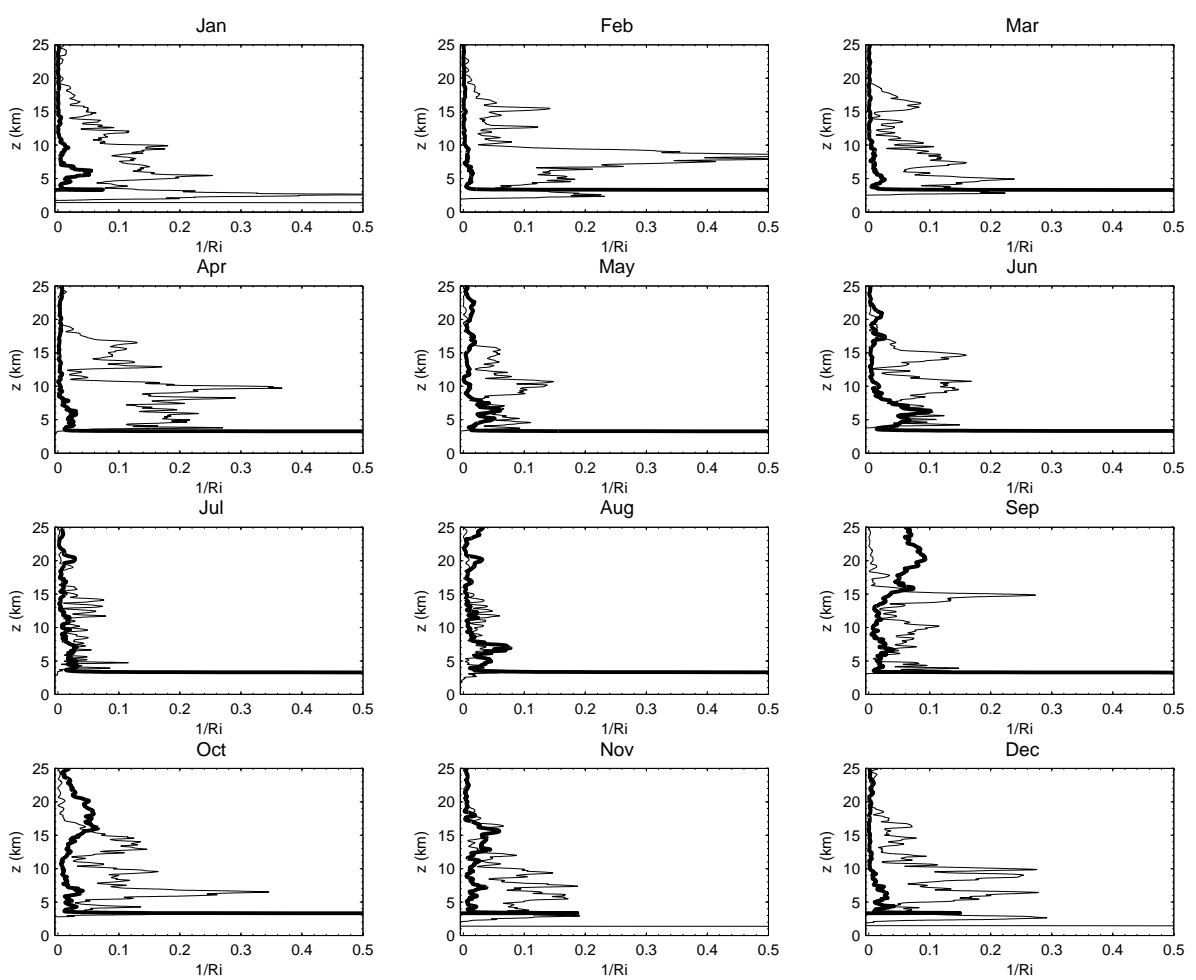

Figure 7. The monthly median for 2005 of the inverse of the Richardson number (1/Ri) for Dome C (thick lines) and Mt. Graham (thin lines).
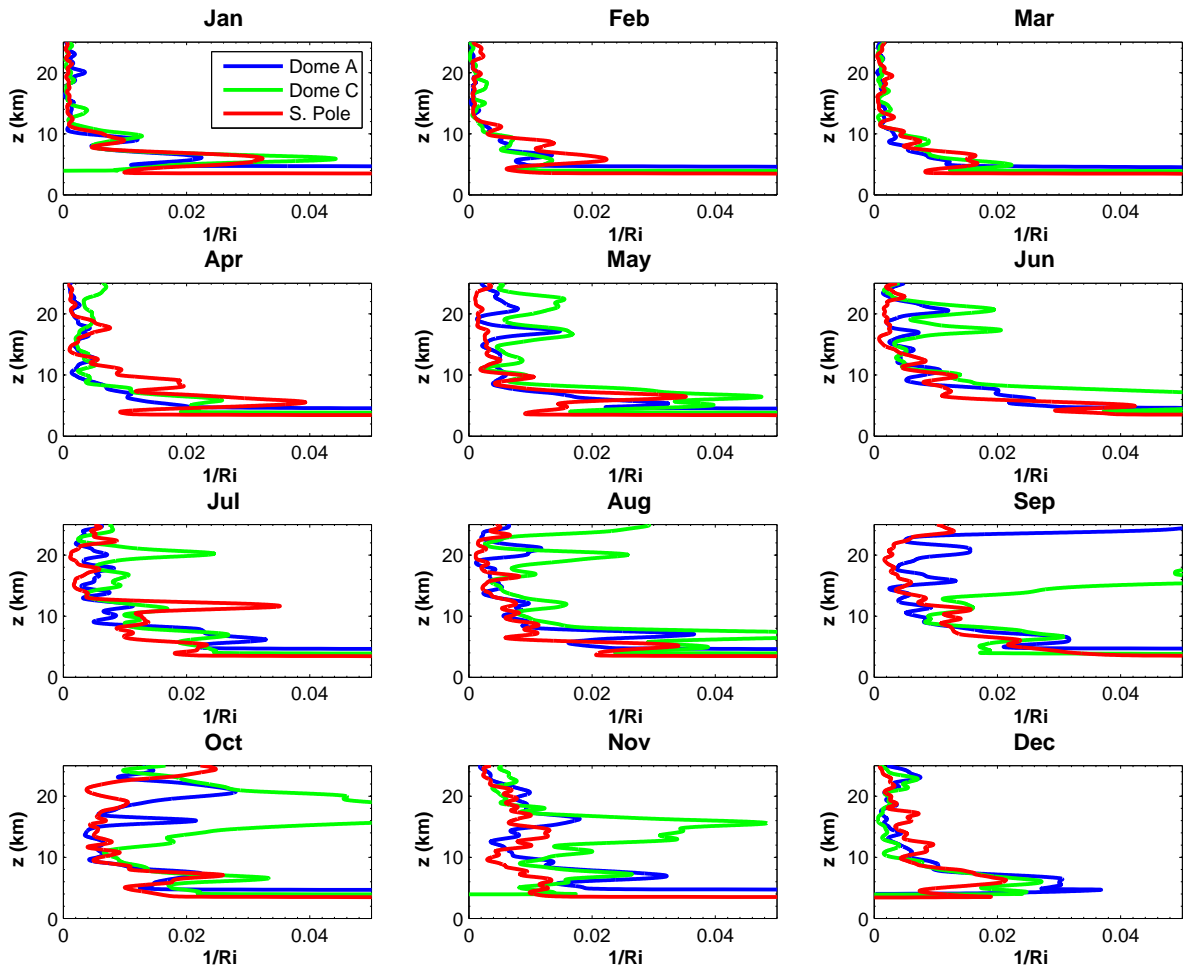

Figure 8. The monthly median for 2005 of the inverse of the Richardson number (1/Ri) for Dome A (blue lines), Dome $\mathrm{C}$ (green lines) and the South Pole (red lines). 
layer needs to be considered with some caution as the ECMWF analyses are not optimized for this layer and radiosoundings are only available for Dome $\mathrm{C}$ and the South Pole. Above Dome A the gradient of the potential temperature is particularly large close to the surface, indicating extreme thermal stability associated to a strong value of the optical turbulence in this layer when a thermodynamic instability occurs. It is even possible that the optical turbulence here is larger than at Dome C. However, our study showed that, to predict the thickness of such a layer it is necessary to have either measurement or simulations made with a mesoscale model with a higher spatial resolution. This is a part of our forthcoming activities.

At present, the real solid argument that makes Dome $\mathrm{C}$ a better place for astronomical applications than the South Pole is the extreme thinness of the optical turbulence surface layer. Dome A probably has comparable or larger optical turbulence values with respect to Dome $\mathrm{C}$ in the surface layer. We cannot conclude whether the surface layer at Dome A is thinner than that observed at Dome C. However our study clearly indicates that Dome $\mathrm{C}$ is not the best site on the Internal Antarctic Plateau with respect to the wind speed (both in the surface layer and in the free atmosphere) nor is it the site with the most stable conditions in the free atmosphere. Both Dome A and the South Pole show more stable conditions.

\section{ACKNOWLEDGMENTS}

This study has been carried out using radiosoundings from the AMRC (Antarctic Meteorological Research Center, University of Wisconsin, Madison ftp://amrc.ssec.wisc.edu/pub/southpole/radiosonde) and from the Progetto di Ricerca 'Osservatorio Meteo Climatologico' of the Programma Nazionale di Ricerche in Antartide (PNRA), http://www.climantartide.it. ECMWF products are extracted from the Catalogue MARS, http://www.ecmwf.int This study has been funded by the Marie Curie Excellence Grant (FOROT) - MEXT-CT-2005-023878.

\section{REFERENCES}

[1] Marks, R., Vernin, J., Azouit, M., Manigault, J., and Clevelin, C., "Measurements of optical seeing on the high antarctic plateau," A\&AS 134, 161-172 (1999).

[2] Lawrence, J., Ashley, M., Tokovinin, A., and Travouillon, T., "Exceptional astronomical seeing conditions above Dome C in Antarctica," Nature 431, 278-280 (2004).

[3] Agabi, A., Aristidi, E., Azouit, M., Fossat, E., Martin, F., Sadibekova, T., Vernin, J., and Ziad, A., "First whole atmosphere nighttime seeing measurements at Dome C, Antarctica," PASP 118, 344-348 (2006).

[4] Marks, R., "Astronomical seeing from the summits of the antarctic plateau," $A \& A$ 385, 328-336 (2002).

[5] Travouillon, T., Ashley, M., Burton, M., Storey, J., and Loewenstein, R., "Atmospheric turbulence at the South Pole and its implications for astronomy," A\&̈A 400, 1163-1172 (2003).

[6] Schwerdtfeger, W., [Weather and climate of the Antarctic, Developements in atmospheric science 15], Elsiever, Amsterdam (1984).

[7] Geissler, K. and Masciadri, E., "Meteorological parameter analysis above Dome C using data from the European Centre for Medium-Range Weather Forecasts," PASP 118, 1048-1065 (2006).

[8] Sadibekova, T., Fossat, E., Genthon, C., Krinner, G., Aristidi, E., Agabi, K., and Azouit, M., "On the atmosphere for astronomers above Dome C, Antarctica," Ant. Scienc. 18 (3), 437-444 (2006).

[9] Hagelin, S., Masciadri, E., Lascaux, F., and Stoesz, J., "Comparison of the atmosphere above the South Pole, Dome C and Dome A: first attempt," MNRAS in press, doi:10.1111/j.1365-2966.2008.13361.x (2008).

[10] Aristidi, E., Agabi, K., Azouit, M., Fossat, E., Vernin, J., Travouillon, T., Lawrence, J., Meyer, C., Storey, J., Halter, B., Roth, W., and Walden, V., "An analysis of temperatures and wind speeds above Dome C, Antarctica," A 6 A 430, 739-746 (2005).

[11] Trinquet, H., Agabi, K., Vernin, J., Azouit, M., Aristidi, E., and Fossat, E., "Nighttime optical turbulence vertical structure above Dome C in Antarctica," PASP 120, 203-211 (2008). 

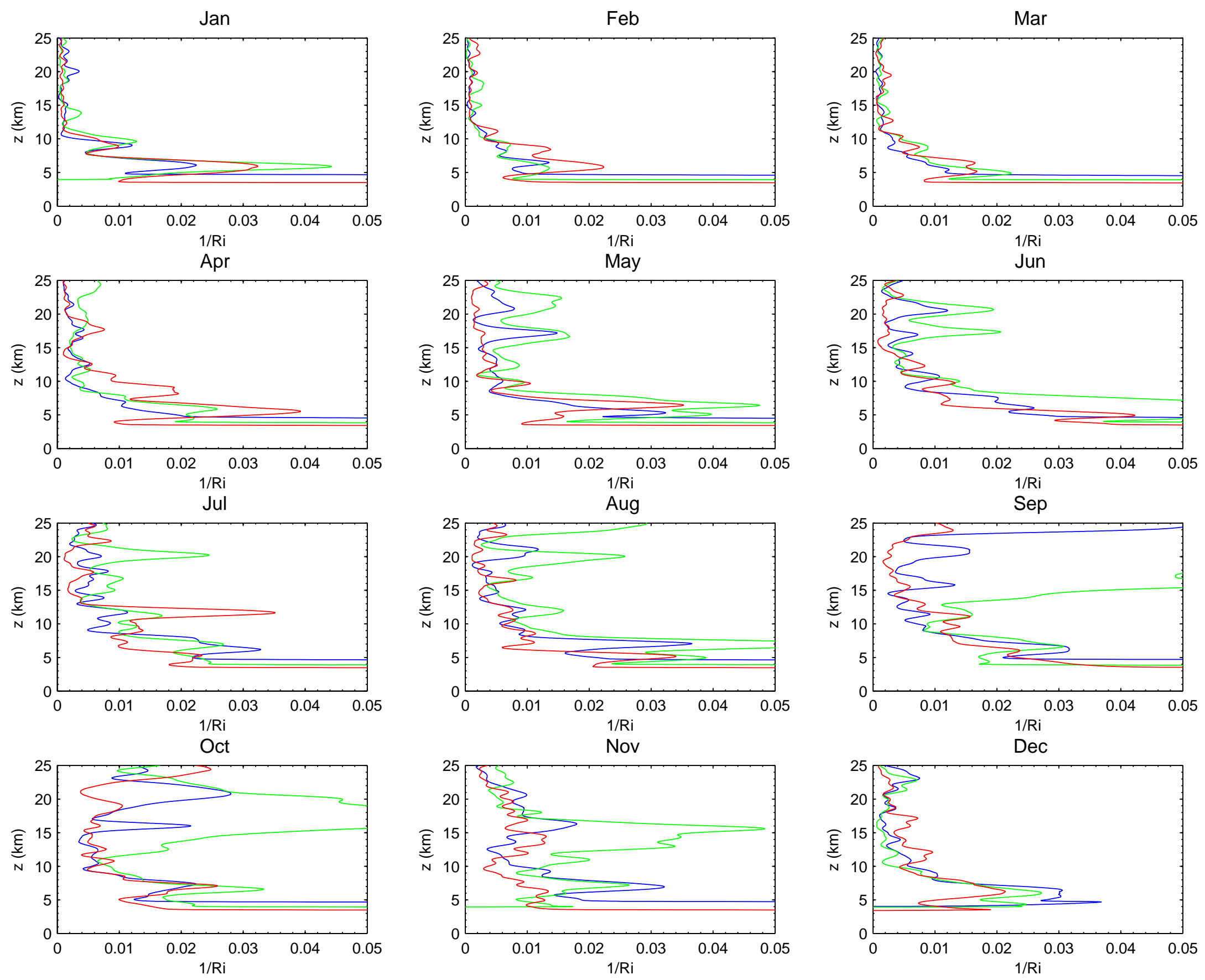